\title{
South Indian leprosy vaccine trial: important lessons for mycobacterial immunology
}

\author{
PAUL E. M. FINE \\ Department of Infectious and Tropical Diseases, London School of \\ Hygiene and Tropical Medicine, Keppel Street, London WC1E 7HT, \\ $U K$
}

Accepted for publication 25 June 1999

Between January 1991 and July 1993, 171,400 individuals living in Chingleput District, South India, were recruited into a major leprosy vaccine trial organized by the Indian Council for Medical Research. The participants were randomized to receive a placebo, a plain BCG vaccine, a combination of BCG plus killed leprosy bacilli, or else a killed environmental mycobacterial vaccine: either 'Mycobacterium W', or 'Mycobacterium ICRC'. Eighty-five percent of these individuals were examined for leprosy between August 1993 and February 1995 and 75\% were examined between January 1997 and September 1998. The codes were broken in December 1998, and revealed that each of the vaccines had provided significant protection over the interval between 4 and 8 years after vaccination: $65.5 \%$ for the ICRC bacillus, 64\% for combined BCG and killed Mycobacterium leprae, 34\% for BCG alone and $25.7 \%$ for the $\mathrm{W}$ bacillus. ${ }^{1}$ These figures, and the data behind them, include several findings of considerable importance for research on mycobacterial immunology and vaccines.

In the first follow-up survey (up to 4 years after vaccination), the incidence of leprosy was higher among each of the active vaccine groups, compared to the placebo recipients. Though these differences were not individually statistically significant, the result is consistent with similar patterns seen in the initial follow up in a leprosy vaccine trial in Burma, ${ }^{2}$ and a tuberculosis vaccine trial in South India. ${ }^{3,4}$ This finding provides important further evidence that antigenic challenge can accelerate progression to mycobacterial disease among individuals either incubating infection at time of vaccination (most likely) or infected very soon after vaccination (less likely explanation). The fact that the negative effect was greater for older (>14 years of age) than younger individuals for recipients of BCG, BCG plus killed M. leprae and Mycobacterium $W$ (this age effect was statistically significant for BCG plus killed $M$. leprae) is consistent with the prior infection interpretation.

In the second follow-up (4-8 years after vaccination), there was significantly less leprosy in each of the active vaccine groups, compared to the controls. The finding of greater protection with the combination BCG plus killed $M$. leprae than with BCG alone is in contrast to findings in Venezuela ${ }^{5}$ and Malawi, ${ }^{6}$ as is the relatively low protection associated with BCG alone. These differences may be added to the long catalogue of variable efficacy results associated with BCG vaccine against leprosy and tuberculosis. ${ }^{7}$ The finding of greater protection with the ICRC than with the $\mathrm{W}$ bacillus is ironic, given that the latter vaccine was 
licensed for therapeutic use in India 1 year ago. It is of interest that this protection appeared to be independent of age. ${ }^{1}$

The observation of highest efficacy attributable to killed ICRC bacillus vaccine has important implications. First is the fact that this protection was induced by a killed vaccine, which is in contrast a view, held widely among mycobacterial vaccinologists, that living vaccines such as BCG are likely to be better than killed vaccines in inducing protective responses. The finding is particularly opportune today, given that much research is devoted to development of new tuberculosis vaccines which might be used in populations with high prevalence of HIV, and thus where live vaccines are likely to be contraindicated. Second is the obvious need to clarify the identity and antigenic nature of the ICRC bacillus, which may explain its appreciable effect on leprosy. This organism has a complicated history, having been isolated originally from leprosy lesions (its isolation was once claimed to be the first successful in vitro culture of $M$. leprae), but later work indicated that it was a relative of M. avium. ${ }^{8}$ If so, this could be of interest, as we now know that $M$. avium and M. leprae share certain antigens (e.g. the $18 \mathrm{kD}^{9}$ ). Third, the published report notes that recipients of the ICRC vaccine had a far higher incidence of 'fluctuant lymphadenitis' compared to recipients of the other vaccines. The pathogenesis of these 'reactions' is of interest (could it be related to prior exposure to a related environmental mycobacterium?), and their association with the most effective vaccine may itself tell us something of the mechanism of the protective immune response.

There are several features of the trial that make interpretation difficult. The authors do not present overall efficacy, combining the two surveys, though this is critical to judging the overall public health utility of these vaccines. In addition, $17.4 \%$ of the trial population had scar evidence of previous BCG vaccination at time of recruitment into this trial. The authors chose to adhere strictly to protocol, and have not separated these individuals (who in effect had received repeat vaccination in the trial) in the analyses. The proportion of individuals with prior scarring was inversely related to age; thus it may be that protection in unvaccinated individuals actually declined with age, as has been observed in several other trials, ${ }^{2,10,11}$ but that this trend is masked by the fact that many younger individuals had already been vaccinated at recruitment. It would not be surprising if the vaccines were less effective in previously vaccinated individuals than in those who had never been vaccinated before. Furthermore, all vaccinations were done in the evenings, avoiding exposure of vaccines to sunlight. This was a clever precaution, given the known sensitivity of BCG and of dermal Langerhans cells to light. However, the absence of a 'daytime control', and the fact that no other trial has used this procedure, makes it impossible to tell if the differences observed between this trial and, for example, the trials of BCG plus killed M. leprae in Venezuela and Malawi, are attributable to evening vaccination or to some other factor. Furthermore, it poses a difficulty in considering the programmatic implementation of the findings, as few vaccination programmes in the world are able to deliver vaccines only in the evening.

Students of mycobacterial vaccines get used to surprises, and this trial is no exception. The investigators are to be commended for the careful implementation of a very major task, and for having contributed importantly to our understanding of mycobacterial immunity. Beyond that, the research community should now seize three major challenges inherent in these results. First, the recognition of protection by a killed organism needs to modify if not replace the dogmatic statements favouring live mycobacterial vaccines in the immunological canon. Second is the need to clarify the nature of the ICRC organism. This should be a straightforward task, given available technology and the current activity in mycobacterial 
genomics (e.g. the completed and imminent sequencing M. tuberculosis ${ }^{12}$ and M. leprae, respectively). Third, there is a very powerful argument to introduce systematic tuberculosis ascertainment as soon as possible into this trial population. This was omitted in the original protocol, perhaps because of disappointment with the negative results of a large BCG trial against tuberculosis conducted earlier in the same region. ${ }^{3}$ However, this population provides an immediate test of the efficacy of four different mycobacterial vaccines against tuberculosis, including two environmental mycobacteria. To neglect this opportunity would be most unfortunate. Funding for such work should have high priority among agencies supporting tuberculosis research.

\section{References}

${ }^{1}$ Gupte MD, Vallishayee RS, Anantharaman DS et al. Comparative leprosy vaccine trial in South India. Ind J Lepr 1998; 70: 369-388.

2 Lwin K, Sundaresan T, Gyi MM et al. BCG vaccination of children against leprosy: fourteen-year findings of the trial in Burma. Bull WHO 1985; 63: 1069-1078.

3 Tuberculosis Prevention Trial Madras. Trial of BCG vaccines in South India for tuberculosis prevention. Ind J Med Res 1980; 72: 1-74.

${ }^{4}$ Springett VH, Sutherland I. Re-examination of the variations in the efficacy of BCG against tuberculosis in clinical trials. Tubercle Lung Disease 1994; 75: 227-233.

5 Convit J, Samson C, Zuniga M et al. Immunoprophylactic trial with combined Mycobacterium leprae/BCG vaccine against leprosy: preliminary results. Lancet 1992; 339: 446-450.

${ }^{6}$ Karonga Prevention Trial Group. Randomized controlled trial of single BCG, repeated BCG, or combined BCG and killed Mycobacterium leprae vaccine for prevention of leprosy and tuberculosis in Malawi. Lancet 1996; 348: 17-24.

${ }^{7}$ Fine PEM. Variation in protection by BCG: implications of and for heterologous immunity. Lancet 1995; 346: 1339-1345.

${ }^{8}$ Bapat CV. Cultivation of Mycobacterium leprae: a new approach. Int J Lepr 1989; 57: 874-879.

9 Booth RJ, Williams DL, Moudgil KD et al. Homologs of Mycobacterium leprae 18-kilodalton and Mycobacterium tuberculosis 19-kilodalton antigens in other mycobacteria. Infect Immun 1993; 61: 1509-1515.

10 Stanley SJ, Howland C, Stone MM, Sutherland I. BCG vaccination of children against leprosy in Uganda: final results. J Hyg (Camb) 1981; 87: 235-248.

11 Leprosy Prevention Trial Madras. BCG prophylaxis for leprosy in South India. Abstracts of the 15th International Leprosy Congress 1998; 99A (Abstract).

12 Cole ST, Brosch R, Parkhill J et al. Deciphering the biology of Mycobacterium tuberculosis from the complete gene sequence. Nature 1998; 393: 537-544. 